ARTICLE

\title{
Subwavelength imaging through ion-beam-induced upconversion
}

Zhaohong $\mathrm{Mi}^{1, \star}$, Yuhai Zhang ${ }^{2, \star}$, Sudheer Kumar Vanga ${ }^{1}$, Ce-Belle Chen ${ }^{1}$, Hong Qi Tan ${ }^{1}$, Frank Watt ${ }^{1}$, Xiaogang Liu ${ }^{2,3,4} \&$ Andrew A. Bettiol ${ }^{1,5}$

The combination of an optical microscope and a luminescent probe plays a pivotal role in biological imaging because it allows for probing subcellular structures. However, the optical resolutions are largely constrained by Abbe's diffraction limit, and the common dye probes often suffer from photobleaching. Here we present a new method for subwavelength imaging by combining lanthanide-doped upconversion nanocrystals with the ionoluminescence imaging technique. We experimentally observed that the ion beam can be used as a new form of excitation source to induce photon upconversion in lanthanide-doped nanocrystals. This approach enables luminescence imaging and simultaneous mapping of cellular structures with a spatial resolution of sub-30 $\mathrm{nm}$.

\footnotetext{
${ }^{1}$ Department of Physics, Centre for Ion Beam Applications, National University of Singapore, Singapore 117542, Singapore. ${ }^{2}$ Department of Chemistry, National University of Singapore, Singapore 117543, Singapore. ${ }^{3}$ Institute of Materials Research and Engineering, Agency for Science, Technology and Research, Singapore 117602, Singapore. ${ }^{4}$ Center for Functional Materials, NUS (Suzhou) Research Institute, Suzhou, Jiangsu 215123, China. ${ }^{5}$ Yale-NUS College, Singapore 138527, Singapore. ${ }^{\star}$ These authors contributed equally to this work. Correspondence and requests for materials should be addressed to F.W. (email: phywattf@nus.edu.sg) or to X.L. (email: chmlx@nus.edu.sg) or to A.A.B. (email: a.bettiol@nus.edu.sg).
} 
P hotoluminescent probes play an indispensable role in labelling and manipulating biological species for many areas of application such as molecular imaging at the subcellular level ${ }^{1-4}$, in vivo biodetection ${ }^{5-8}$ and targeted intracellular delivery of therapeutics 9,10 In particular, lanthanide-doped upconversion nanocrystals ${ }^{11-13}$ have recently gained considerable attention for use as biomarkers owing to their unique ability to convert low-energy light into high-energy photons, coupled with the absence of photobleaching and photoblinking ${ }^{14,15}$. However, an infrared laser, either in continuous- or pulsed-wave mode, is generally needed to implement photon upconversion ${ }^{16-20}$. The use of the laser as the excitation source inevitably imposes an inherent constraint for high-resolution imaging because of Abbe's diffraction limit.

It has been well established that hexagonal-phase $\mathrm{NaYF}_{4}$ is one of the most efficient host materials frequently utilized for preparing upconversion nanocrystals ${ }^{12,21}$. The upconversion nanocrystals are typically doped with ytterbium $\left(\mathrm{Yb}^{3+}\right)$ sensitizer ions, which absorb infrared radiation centring at $980 \mathrm{~nm}$ and non-radiatively transfer their absorption to activator ions such as thulium $\left(\mathrm{Tm}^{3+}\right)$, erbium $\left(\mathrm{Er}^{3+}\right)$ or holmium $\left(\mathrm{Ho}^{3+}\right)$. The notable prospects of lanthanide-doped nanocrystals, including non-photobleaching, tunable emission wavelength and controllable particle size ${ }^{12,14}$, have provided new opportunities for bioimaging applications in a variety of research fields ${ }^{6,7,22}$. In addition, the excitation of these nanocrystals in the near-infrared region eliminates background autofluorescence ${ }^{6,8}$. However, these imaging studies have been unable to provide detailed information on the single-particle level owing to the diffraction limit of infrared excitation light associated with conventional or even confocal microscope setups ${ }^{23,24}$.

To overcome the diffraction limit, a variety of optical superresolution techniques, for instance stimulated-emission depletion microscopy ${ }^{25}$, have been developed. Alternatives to these superresolution techniques are methods involving charged particles such as electron or ion beams, with the benefit of rendering much shorter de Broglie wavelengths. For example, electron microscope-based cathodoluminescence has been successfully utilized for high-resolution luminescence imaging ${ }^{26,27}$. However, the electrons suffer from large angle scattering when interacting with biological samples, which in turn compromises the resolution, especially for tissue imaging at a substantial depth.

Here we report, for the first time, the observation of photon upconversion through excitation of lanthanide-doped nanocrystals under a beam of helium ions. The use of mega-electron-volt- (MeV) focused helium ions offer significant advantages, as they can penetrate much thicker biological samples (up to several microns) with very little deviation in their trajectories $^{28}$ (see Supplementary Fig. 1). We thus reason that the $\mathrm{MeV}$-focused ion beam may serve as a new form of excitation source to induce photon upconversion in lanthanide-doped nanocrystals and, more importantly, to allow for high-resolution luminescence imaging beyond the diffraction limit.

\section{Results}

Construction of ion-beam imaging setup. The basic experimental setup is shown in Fig. 1a. A beam of $1.6 \mathrm{MeV}$ helium ions ( $\alpha$-particles) is produced by a Singletron ion accelerator. A sample comprising $\mathrm{NaYF}_{4}: \mathrm{Yb} / \mathrm{Tm}$ nanorods is placed in a vacuum chamber $\left(10^{-6} \mathrm{mbar}\right)$ at a position situated exactly along the beam path. A customized double-piece parabolic mirror with front and rear openings is used to collect emission photons induced by the ion beam and, concurrently, allow the ion beam to pass through the mirror (Supplementary Fig. 1).
The convergent lens-coupled parabolic mirror allows the emitted light to be focused into a fibre, which guides the light out of the vacuum chamber. The emitted photons are then captured either by a photomultiplier tube for luminescence imaging or by a spectrometer for spectroscopic characterization. A Si surface barrier detector is used to perform scanning transmission ion microscopy imaging by measuring the energy loss during the penetration of the ions into a given sample 28,29 .

The inelastic collision of helium ions with atomic electrons in a crystal can lead to energy loss dominated through an excitation and atomic ionization process ${ }^{30}$. To understand the efficacy of the ionization in the $\mathrm{NaYF}_{4}: \mathrm{Yb} / \mathrm{Tm}$ nanocrystal for photon upconversion, we first performed simulations on the energy distribution of the ionized secondary electrons using a HansenKocbach-Stolterfoht theoretical model (Supplementary Notes) ${ }^{30}$. Our simulation result shows that the ionized electrons with energies larger than $1.265 \mathrm{eV}$ (equivalent to $980 \mathrm{~nm}$ ) hold a large portion (estimated to be $97.5 \%$ ) of the total cross-sections (Fig. 1b). Thus, the ionized electrons within this energy portion can potentially be utilized by the $\mathrm{Yb} / \mathrm{Tm}$ co-doped nanocrystal. On the basis of the energy-matching principle, we propose an energy transfer mechanism that governs the photon upconversion in the $\mathrm{NaYF}_{4}: \mathrm{Yb} / \mathrm{Tm}$ nanocrystal system (Fig. 1c and Supplementary Fig. 2). It should be pointed out that the ionized electrons with energies higher than $1.265 \mathrm{eV}$ may partially lose their energy by ionization, collision or phononcoupling processes to match the energy levels of $\mathrm{Yb}^{3+}$ or $\mathrm{Tm}^{3+}$ for effective upconversion pumping.

Spectroscopic study of lanthanide-doped crystals. To validate our hypothesis, we prepared a set of $\mathrm{NaYF}_{4}$-based nanorods with different dopant compositions through a hydrothermal procedure $^{23}$ and systematically investigated their response to $\alpha$-particle irradiation. Scanning electron microscopic imaging revealed the formation of monodisperse nanorods with an average size of $1.9 \mu \mathrm{m} \times 150 \mathrm{~nm}$ (Fig. 2a and Supplementary Figs 3 and 4$)$. When singly-doped with $\mathrm{Yb}^{3+}(60 \mathrm{~mol} \%)$ as the activator, the nanorods gave rise to emission at $975 \mathrm{~nm}$ on $\alpha$-particle excitation, corresponding to ${ }^{2} \mathrm{~F}_{5 / 2} \rightarrow{ }^{2} \mathrm{~F}_{7 / 2}$ transition of $\mathrm{Yb}^{3+}$ (Fig. 2b). In contrast, $\mathrm{NaYF}_{4}: \mathrm{Tm}(2 \mathrm{~mol} \%)$ nanorods exhibited an intense emission at $800 \mathrm{~nm}$ and two weak emissions at 450 and $480 \mathrm{~nm}$, corresponding to ${ }^{3} \mathrm{H}_{4} \rightarrow{ }^{3} \mathrm{H}_{6},{ }^{1} \mathrm{D}_{2} \rightarrow{ }^{3} \mathrm{~F}_{4}$ and ${ }^{1} \mathrm{G}_{4} \rightarrow{ }^{3} \mathrm{H}_{6}$ optical transitions of $\mathrm{Tm}^{3+}$, respectively. These results clearly verify that both $\mathrm{Yb}^{3+}$ and $\mathrm{Tm}^{3+}$ ions can directly harvest the energy of the ionized electrons upon the excitation with the $\alpha$-particles. Intriguingly, in the case of $\mathrm{NaYF}_{4}$ nanorods co-doped with $\mathrm{Yb} / \mathrm{Tm}(60 / 2 \mathrm{~mol} \%)$, the blue emissions at 450 and $480 \mathrm{~nm}$ of $\mathrm{Tm}^{3+}$ showed a considerable enhancement, suggesting that the addition of $\mathrm{Yb}^{3+}$ in the $\mathrm{NaYF}_{4}: \mathrm{Tm}$ nanorods promotes ion-beam-induced upconversion emission of $\mathrm{Tm}^{3+}$ at short wavelengths through energy transfer upconversion.

To shed more light on the energy transfer between $\mathrm{Yb}^{3+}$ and $\mathrm{Tm}^{3+}$, we prepared a series of $\mathrm{NaYF}_{4}: \mathrm{Yb} / \mathrm{Tm}$ nanorods with varied $\mathrm{Yb}^{3}+$ doping concentrations (10-98 mol\%). We collected their luminescence spectra (Supplementary Figs 5 and 6) under $\alpha$-particle irradiation and integrated the overall emission intensity for $\mathrm{Tm}^{3+}$ and $\mathrm{Yb}^{3+}$ ions, respectively. The intensity ratios of $I_{\mathrm{Tm}} / I_{\mathrm{Yb}}$, plotted against $\mathrm{Yb}$ doping content, was used to show the relative intensity change in $\mathrm{Tm}^{3+}$ and $\mathrm{Yb}^{3+}$ emissions. As shown in Fig. 2c, the measured intensity ratio of $I_{\mathrm{Tm}} / I_{\mathrm{Yb}}$ increased from 3.3 to 10.2 with the increase in $\mathrm{Yb}^{3+}$ concentration from 10 to $50 \mathrm{~mol} \%$ and then decreased to 3.3 at a $\mathrm{Yb}^{3}+$ concentration of $98 \mathrm{~mol} \%$. Such inverse-parabolic profile provides a strong evidence for the energy transfer between $\mathrm{Yb}^{3+}$ and $\mathrm{Tm}^{3+}$. Particularly, the rising stage of $I_{\mathrm{Tm}} / I_{\mathrm{Yb}}$ indicates the occurrence of 

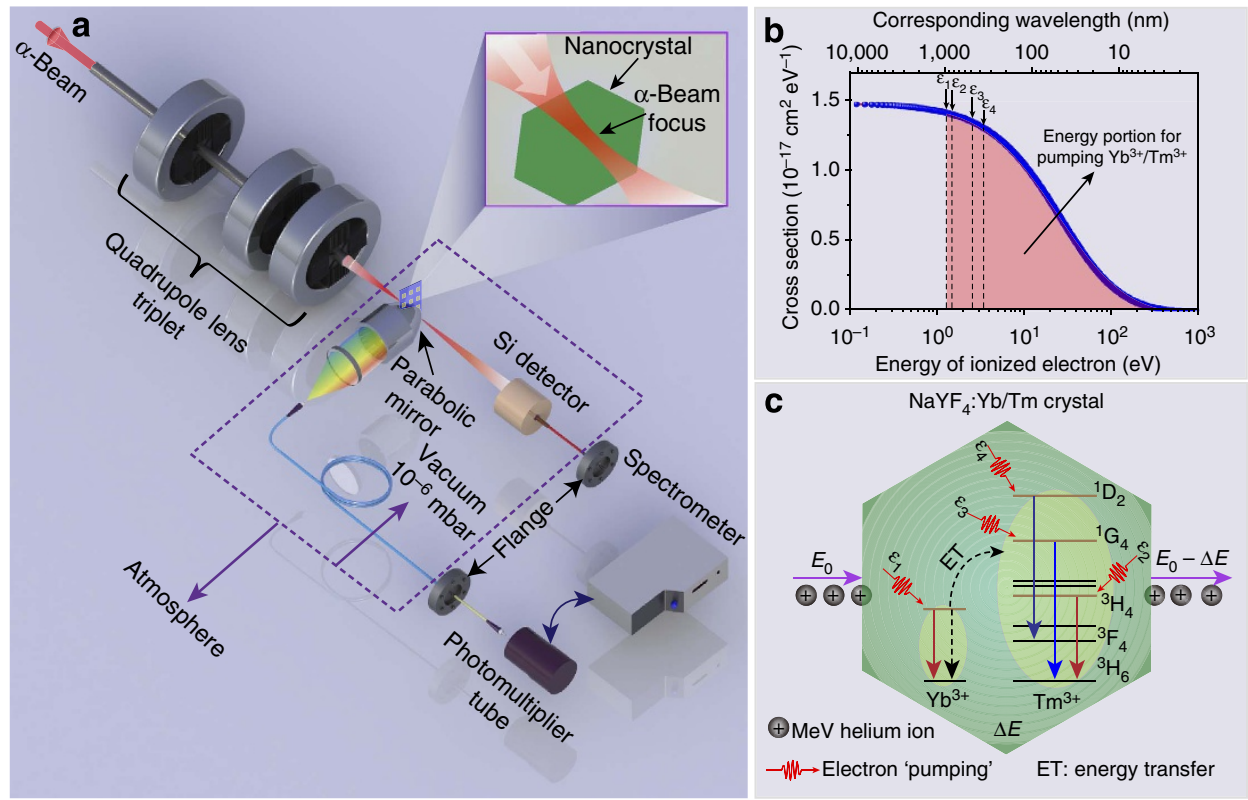

Figure 1 | Experimental setup and proposed ionoluminescence mechanism. (a) Artist's view of the basic experimental setup. The focused beam with a spot size of sub-30 nm features can be achieved using a spaced triplet of compact magnetic quadrupole lenses. A Si surface barrier detector is equipped for measuring the energy loss distribution of the ions. (b) Calculated energy distribution of the ionized electrons by bombarding the MeV $\alpha$-particles on the lanthanide-doped nanocrystals, showing different cross-sections of the resulting electrons at specific energies. Note that most of the ionized electrons have energies mainly located in the visible and infrared spectral region. (c) Proposed upconversion mechanism under $\alpha$-beam irradiation. The incident helium ions with energy of $E_{0}$ deposit a certain amount of energy $(\Delta E)$ onto the crystal to cause the atomic ionization inside the crystal. Subsequently, the ionized secondary electrons can release their energy, most likely during the electron-hole recombination process and successively transfer the energy to $\mathrm{Yb}^{3+}$ and $\mathrm{Tm}^{3+}$. An energy transfer from the excited $\mathrm{Yb}^{3+}$ to its neighbouring $\mathrm{Tm}^{3+}$ ions then populates the excited states (for example, ${ }^{3} \mathrm{H}_{4},{ }^{1} \mathrm{G}_{4}$ and ${ }^{1} \mathrm{D}_{2}$ ) of $\mathrm{Tm}^{3+}$.
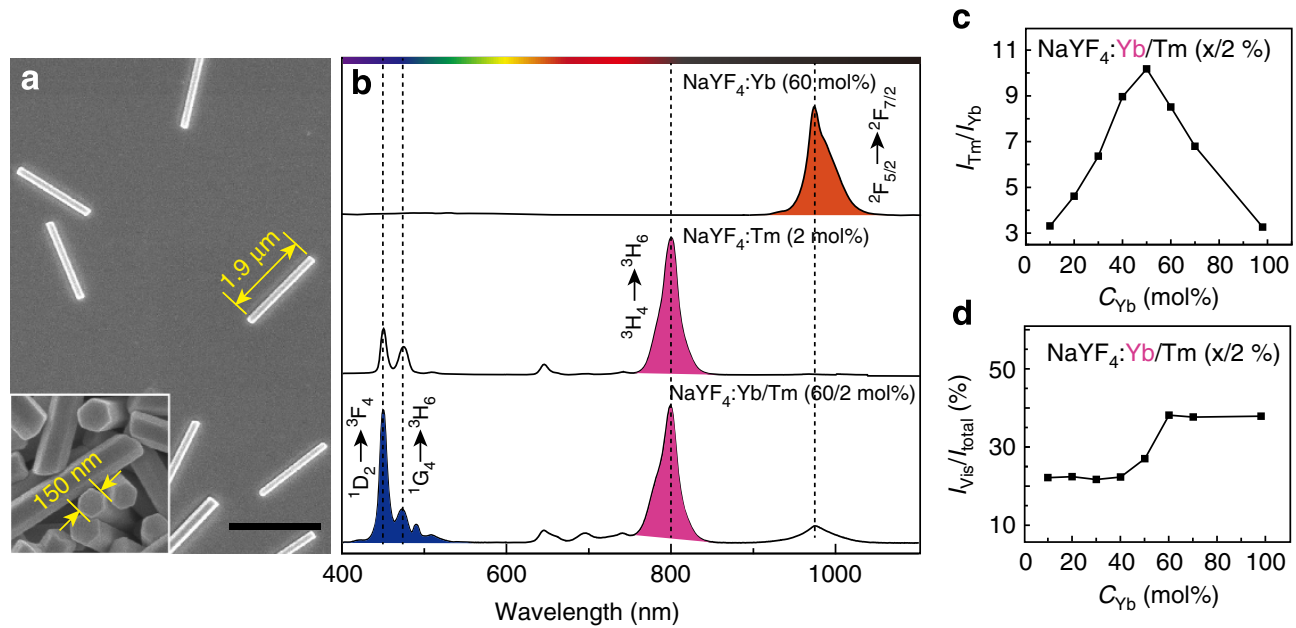

Figure 2 | Spectroscopic analysis of ionoluminescence. (a) Scanning electron microscopy (SEM) image of the as-synthesized $\mathrm{Yb}^{3+} / \mathrm{Tm}^{3+}-\mathrm{co}^{-\mathrm{doped}}$ $\mathrm{NaYF}_{4}$ nanorods under investigation. The inserted high-resolution SEM image shows hexagonal cross-sections of these nanorods. Scale bar, $2 \mu \mathrm{m}$. (b) Comparative emission spectra of the $\mathrm{NaYF}_{4}$-based nanorods with different dopant compositions when irradiated with $\alpha$-particles. (c) The plot of the emission ratio of $\mathrm{Tm}^{3+}$ and $\mathrm{Yb}^{3+}\left(I_{\mathrm{Tm}} / I_{\mathrm{Yb}}\right)$ as a function of $\mathrm{Yb}^{3+}$ doping concentration, supporting the energy transfer between the two lanthanide ions. (d) Optimization of $\mathrm{Yb}^{3+}$ doping concentration for maximal emission output in the visible range. The ratio of $I_{\mathrm{V} \text { is }} / I_{\text {total }}$ represents the percentage of integrated visible emission in the total emission covering the range from 350 to 1,100 nm.

efficient energy transfer from $\mathrm{Yb}^{3+}$ to $\mathrm{Tm}^{3+}$, thus resulting in the pronounced enhancement of upconversion emission in short wavelengths. The descending stage of $I_{\mathrm{Tm}} / I_{\mathrm{Yb}}$ can be ascribed to the back-energy-transfer from $\mathrm{Tm}^{3+}$ to $\mathrm{Yb}^{3+}$ at high $\mathrm{Yb}^{3+}$ concentrations, analogous to the scenario in photon upconversion process in which a $980 \mathrm{~nm}$ laser (Supplementary Fig. 7 and Supplementary Table 1) is employed as the excitation source $^{23}$.
Luminescence imaging. High-resolution imaging can be achieved through $\alpha$-beam irradiation of lanthanide-doped nanomaterials because the spot size of $\alpha$-beam can be readily focused down to sub-30 nm (refs 28,29). Considering that the spectral-response range of the photodetector used falls within the visible spectrum, we have adopted $\mathrm{Yb}^{3+} / \mathrm{Tm}^{3+}(60 / 2 \mathrm{~mol} \%)$ as the optimal combination for maximal visible emission (Fig. $2 \mathrm{~d}$ and Supplementary Fig. 6). Images of the $\mathrm{NaYF}_{4}: \mathrm{Yb} / \mathrm{Tm}$ 

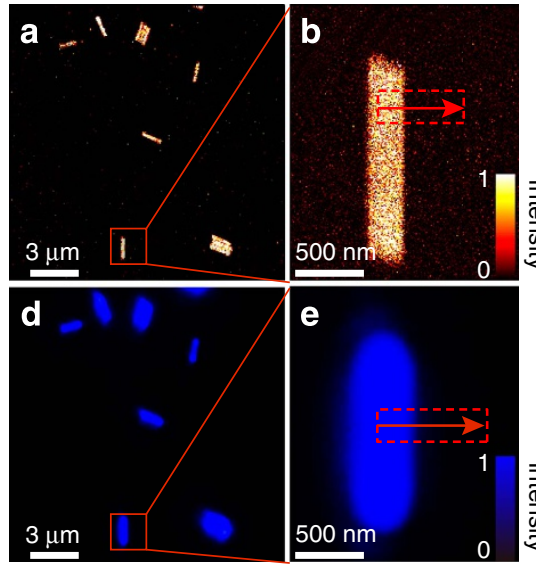

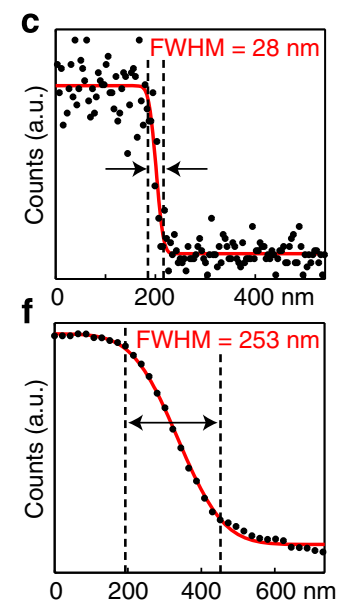

g

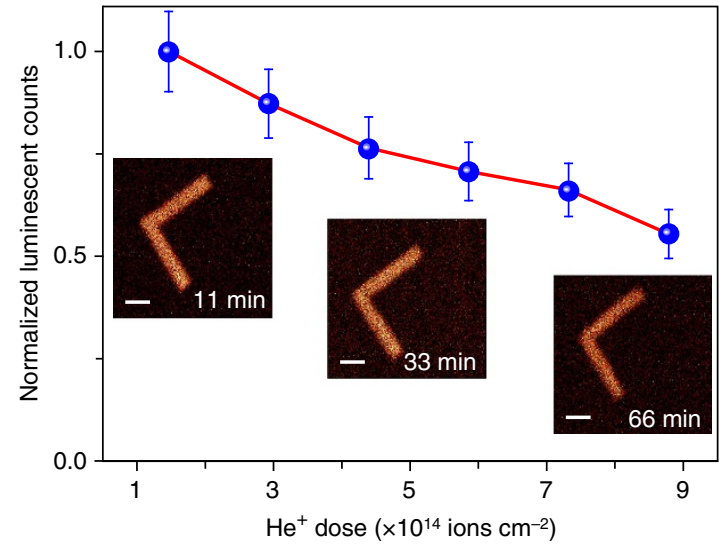

Figure 3 | Luminescence imaging of $\mathrm{NaYF}_{\mathbf{4}}: \mathbf{Y b} / \mathbf{T m}(\mathbf{6 0 / 2}$ mol\%) nanorods. (a) lonoluminescence image of the as-synthesized nanorods through $\alpha$-particle excitation. (b) High-magnification ionoluminescence image of a single nanorod as marked in $\mathbf{a}$. (c) The corresponding line-scanning profile extracted from the intensity counting at the region marked in $\mathbf{b}$ along the arrow, indicating an imaging resolution of about $28 \mathrm{~nm}$. (d) Photoluminescence image of the same sample taken by using $980 \mathrm{~nm}$ laser excitation. (e) High-magnification photoluminescence image of the same nanorod as shown in $\mathbf{b}$. (f) The corresponding line-scanning profile from the image shown in e showing a diffraction-limited resolution of $253 \mathrm{~nm}$ associated with conventional upconversion microscopes. ( $(\mathbf{g})$ lonoluminescence intensity profile as a function of the accumulated dosage of helium ions showing the considerable iono-bleaching resistance of the nanorods. The inserted images, taken at different time intervals (11, 33 and 66 min), indicate that the emission brightness of the nanorods remains essentially unaltered over time. Scale bars, $500 \mathrm{~nm}$. The error bar represents the standard deviation of luminescence counts obtained from a single nanorod in two separate measurements.
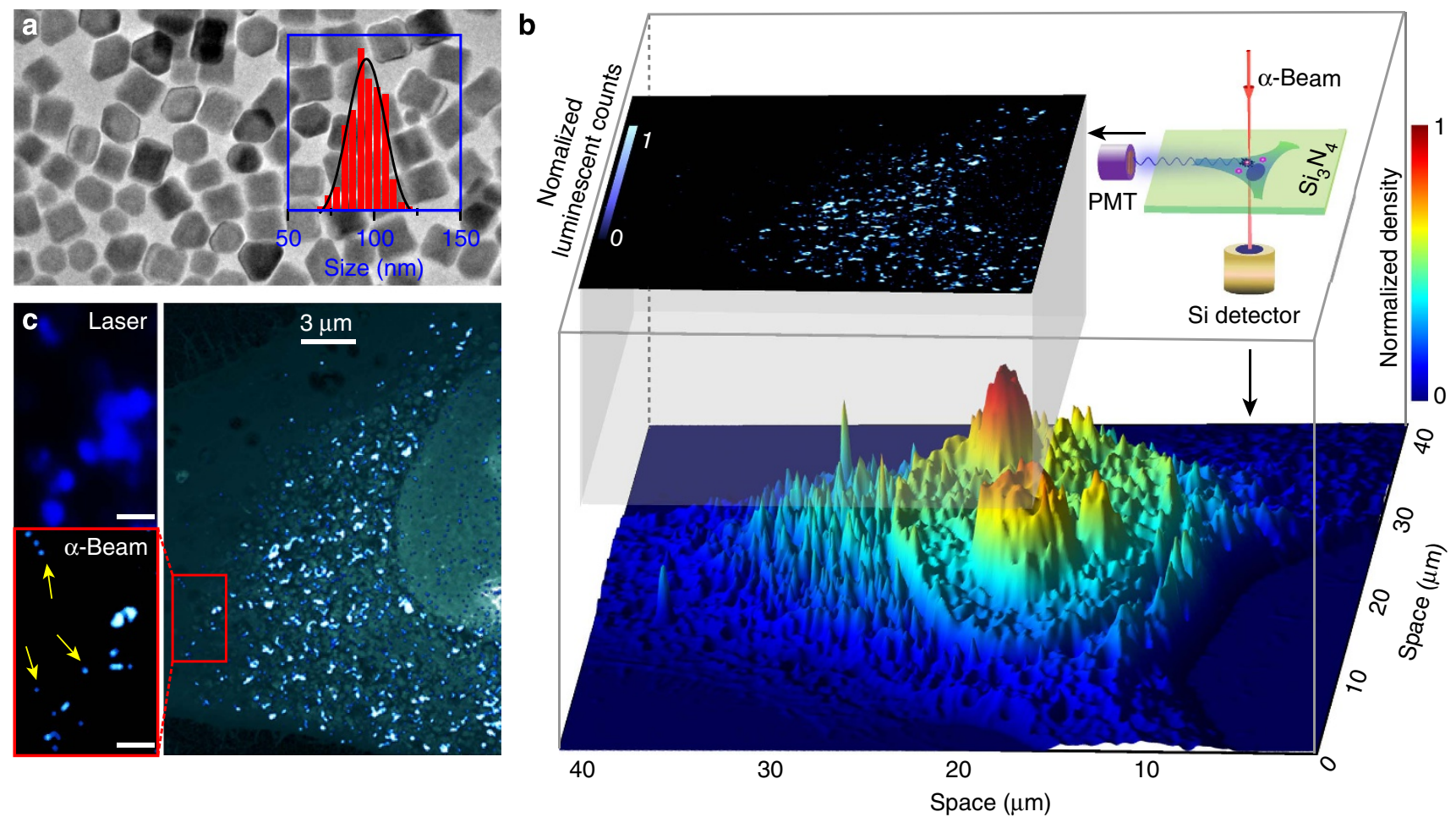

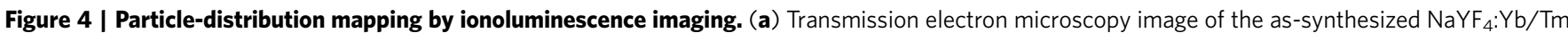
$(60 / 2 \mathrm{~mol} \%)$ nanoparticles. The inserted histogram shows the size distribution of these nanoparticles. (b) Basic experimental design for structural determination and ionoluminescence imaging of the HeLa cell after uptake of the nanoparticles. The luminescence mapping of the nanoparticles and the 3D rendering of detailed cellular structures can be simultaneously implemented by capturing $\alpha$-particle-induced photons through a photomultiplier tube (PMT) and by scanning transmission ion microscopy, respectively. (c) Comparative photoluminescence (top left) and ionoluminescence (right) imaging, with the latter clearly showing the ability to resolve single nanoparticles (marked by the arrows as shown in the magnified image). Note that top-left and bottom-left images are taken from the same section of the cell. Scale bars, $1 \mu \mathrm{m}$. Note that the photoluminescence image was generated by using a 980 -nm-diode laser (Supplementary Fig. 12). 
$(60 / 2 \mathrm{~mol} \%)$ nanorods were recorded in a $512 \times 512$ pixel array at a count rate of around 15,000 helium ions per second by detecting the $\alpha$-particle-induced luminescence (Fig. 3a,b). To ascertain the spatial resolution of the ionoluminescence image, a representative line-scanning profile of an individual nanorod was collected and presented in Fig. 3c. By fitting the profile using a modified Gaussian model ${ }^{31}$, the imaging resolution of the $\alpha$-particle-based ionoluminescence technique was determined to be $28 \mathrm{~nm}$ as defined by full-width at half maximum. By comparison, conventional optical microscopies equipped with a 980-nm diode laser showed a resolution limit of $\sim 253 \mathrm{~nm}$ (Fig. $3 \mathrm{~d}-\mathrm{f}$ and Supplementary Fig. 8). It should be noted that the effect of iono-bleaching, typically associated with the reduction in emission intensity in dye- or quantum dot-based systems ${ }^{32,33}$, does not pose a constraint to lanthanide-doped nanomaterials (Fig. 3g and Supplementary Figs 9 and 10).

\section{Discussion}

Importantly, the combination of scanning transmission ion microscopy and the $\alpha$-particle-induced luminescence technique enables simultaneous structural determination and luminescence imaging on a single-cell level. As a proof of concept, we prepared $\mathrm{NaYF}_{4}: \mathrm{Yb} / \mathrm{Tm}(60 / 2 \%)$ nanoparticles $(\sim 95 \mathrm{~nm})$ and incubated them with Human cervical carcinoma cells, which were seeded on a 100-nm-thick silicon-nitride membrane (Fig. 4a,b and Supplementary Fig. 11). By detecting the energy loss of transmitted ions through a $\mathrm{Si}$ surface barrier detector, we were able to generate an areal density map of a whole HeLa cell by scanning transmission ion microscopy, which provides detailed information on cellular structures (Fig. 4b). Concurrently, $\alpha$-particle-induced photons were captured by a photomultiplier tube for luminescence mapping of the nanoparticles (Fig. 4b). The coupling of $\alpha$-particle-induced luminescence imaging with scanning transmission ion microscopy allowed us to precisely locate the nanoparticles within the whole cell (Fig. 4c). Remarkably, single nanoparticles after cellular internalization could be resolved by our technique (see the enlarged panel in Fig. 4c). This was in stark contrast with the limit of resolution achievable by a conventional microscope equipped with a $980-\mathrm{nm}$ diode laser (Fig. 4c, top-left panel). In the latter case, the photoluminescence imaging from the same area showed much reduced resolution (Supplementary Fig. 12).

Our findings could influence the study of the dynamics of upconversion processes and provide a better understanding of energy transfer in lanthanide-doped materials systems where the source of excitation may play a crucial role. The results presented here suggest that a sub-30 nm imaging resolution for upconversion nanocrystals is achievable through the use of $\alpha$-particle-induced secondary electrons. By combining upconversion luminescence with scanning transmission ion microscopy, we have been able to map the distribution of individual nanoparticles within a whole cell and simultaneously reveal the $3 \mathrm{D}$ cellular structure at ultrahigh spatial resolution. This methodology will enable important applications in probing biological and biomedical processes at the subcellular level, for example, the quantitative measurement of intracellular bio-distribution of drugs delivered by upconversion nanoparticles ${ }^{34,35}$.

\footnotetext{
Methods

Preparation of upconversion nanocrystals. Lanthanide-doped nanorods and nanoparticles were prepared through a hydrothermal method ${ }^{23}$ and a coprecipitation method ${ }^{36}$, respectively. The as-prepared nanocrystals were washed with $\mathrm{HCl}$ to remove oleic acid molecules that were used as surface-capping ligands during the synthesis. Detailed experimental procedures are provided in the Supplementary Methods.
}

Preparation of cells. Human cervical carcinoma cells were seeded onto 100 -nm-thick silicon-nitride membranes at a density of 9,000 cells $\mathrm{cm}^{-2}$ in Dulbecco's Modified Eagle's medium containing fetal bovine serum (10\%),

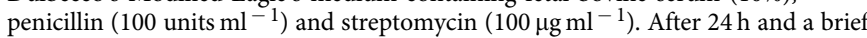
wash with phosphate-buffered saline, the cells were incubated in complete medium containing the as-synthesized $\mathrm{NaYF}_{4}: \mathrm{Yb} / \mathrm{Tm}(60 / 2 \%)$ nanoparticles $\left(10 \mu \mathrm{g} \mathrm{ml}^{-1}\right)$ for another $24 \mathrm{~h}$. Following another wash with Hepes-buffered saline, the particle-treated cells were then transferred to a solution of $2 \%$ glutaraldehyde and stored overnight prior to intermediate dehydration using an increasing ethanol gradient. Complete dehydration was then achieved by critical-point drying.

Instrumentation and imaging. Photoluminescence images were taken by an Olympus BX51 optical microscope equipped with a $980-\mathrm{nm}$ diode laser. The $\alpha$-particle-induced luminescence imaging was achieved by collecting the luminescence photons with a customized double-piece parabolic mirror. The collected photons were then detected by a Hamamatsu photomultiplier tube (PMT) $\mathrm{R} 7400 \mathrm{P}$ equipped with the photon counting unit C9744. The data were collected and processed using the IONDAQ data acquisition system ${ }^{37}$ to generate the ionoluminescence images (Supplementary Fig. 13). The energy loss of an ion transmitted through a sample depends on the sample composition and thickness. Thus, the areal density can be expressed by equation (1):

$$
\text { Areal density }=\int_{E_{0}}^{E_{r}}\left[\frac{d E}{d(\rho z)}\right]^{-1} d E
$$

where $E_{0}$ is the initial ion energy, $E_{r}$ is the remaining energy of the ion after passing through the sample, and $\rho=\rho(z)$ is the mass density of the sample at a depth of $z$. Through scanning transmission ion microscopy, the transmitted ion energies and number of ions at each pixel within the scanned area can be measured by a $\mathrm{Si}$ surface barrier detector to render the areal density map.

\section{References}

1. Fernández-Suárez, M. \& Ting, A. Y. Fluorescent probes for super-resolution imaging in living cells. Nat. Rev. Mol. Cell Biol. 9, 929-943 (2008).

2. Ueno, T. \& Nagano, T. Fluorescent probes for sensing and imaging. Nat. Methods 8, 642-645 (2011).

3. Haase, M. \& Schäfer, H. Upconverting nanoparticles. Angew. Chem. Int. Ed. 50, 5808-5829 (2011).

4. Bünzli, J. C. G. \& Piguet, C. Taking advantage of luminescent lanthanide ions Chem. Soc. Rev. 34, 1048-1077 (2005).

5. Vetrone, F., Boyer, J. C., Capobianco., J. A., Speghini, A. \& Bettinelli, M. Significance of $\mathrm{Yb}^{3}+$ concentration on the upconversion mechanisms in codoped $\mathrm{Y}_{2} \mathrm{O}_{3}: \mathrm{Er}^{3+}, \mathrm{Yb}^{3+}$ nanocrystals. J. Appl. Phys. 96, 661-667 (2004).

6. Zhou, J., Liu, Q., Feng, W., Sun, Y. \& Li, F. Upconversion luminescent materials: advances and applications. Chem. Rev. 115, 395-465 (2014).

7. Zheng, Q. et al. Frequency-upconverted stimulated emission by simultaneous five-photon absorption. Nat. Photon. 7, 234-239 (2013).

8. Chen, G., Qiu, H., Prasad, P. N. \& Chen, X. Upconversion nanoparticles: design, nanochemistry, and applications in theranostics. Chem. Rev. 114, 5161-5214 (2014).

9. Dai, Y. et al. In vivo multimodality imaging and cancer therapy by near-infrared light-triggered trans-platinum pro-drug-conjugated upconverison nanoparticles. J. Am. Chem. Soc. 135, 18920-18929 (2013).

10. Zou, W., Visser, C., Maduro, J. A., Pshenichnikov, M. S. \& Hummelen, J. C. Broadband dye-sensitized upconversion of near-infrared light. Nat. Photon. 6, 560-564 (2012).

11. Auzel, F. Upconversion and anti-Stokes processes with $\mathrm{f}$ and $\mathrm{d}$ ions in solids. Chem. Rev. 104, 139-173 (2004).

12. Wang, F. et al. Simultaneous phase and size control of upconversion nanocrystals through lanthanide doping. Nature 463, 1061-1065 (2010).

13. Gai, S., Li, C., Yang, P. \& Lin, J. Recent progress in rare earth micro/ nanocrystals: soft chemical synthesis, luminescent properties, and biomedical applications. Chem. Rev. 114, 2343-2389 (2014).

14. Wu, S. et al. Non-blinking and photostable upconverted luminescence from single lanthanide-doped nanocrystals. Proc. Natl Acad. Sci. USA 106, 10917-10921 (2009).

15. Schietinger, S. et al. Plasmon-enhanced upconversion in single $\mathrm{NaYF}_{4}: \mathrm{Yb}^{3+} / \mathrm{Er}^{3+}$ codoped nanocrystals. Nano Lett. 10, 134-138 (2010).

16. Cheng, Y. Y. et al. On the efficiency limit of triplet-triplet annihilation for photochemical upconversion. Phys. Chem. Chem. Phys. 12, 66-71 (2010).

17. Zhao, J. et al. Single-nanocrystal sensitivity achieved by enhanced upconversion luminescence. Nat. Nanotech. 8, 729-734 (2013).

18. Wang, J. et al. Photon energy upconversion through thermal radiation with the power efficiency reaching 16\%. Nat. Commun. 5, 5669 (2014).

19. Liu, X. et al. Probing the nature of upconversion nanocrystals: instrumentation matters. Chem. Soc. Rev. 44, 1479-1508 (2015).

20. Deng, R. et al. Temporal full-colour tuning through non-steady-state upconversion. Nat. Nanotech. 10, 237-242 (2015). 
21. Gargas, D. J. et al. Engineering bright sub-10-nm upconverting nanocrystals for single-molecule imaging. Nat. Nanotech. 9, 300-305 (2014).

22. Lu, Y. et al. Tunable lifetime multiplexing using luminescent nanocrystals. Nat. Photon. 8, 32-36 (2014).

23. Zhang, Y. et al. Multicolor barcoding in a single upconversion crystal. J. Am. Chem. Soc. 136, 4893-4896 (2014).

24. Caillat, L. et al. Multiphoton upconversion in rare earth doped nanocrystals for sub-diffractive microscopy. Appl. Phys. Lett. 102, 143114 (2013).

25. Rittweger, E., Han, K. Y., Irvine, S. E., Eggeling, C. \& Hell, S. W. STED microscopy reveals crystal colour centres with nanometric resolution. Nat. Photon. 3, 144-147 (2009).

26. Glenn, D. R. et al. Correlative light and electron microscopy using cathodoluminescence from nanoparticles with distinguishable colours. Sci. Rep. 2, 865 (2012)

27. Furukawa, T. et al. High-resolution microscopy for biological specimens via cathodoluminescence of Eu- and $\mathrm{Zn}$-doped $\mathrm{Y}_{2} \mathrm{O}_{3}$ nanophosphors. Opt. Express 21, 25655-25663 (2013).

28. Chen, X. et al. Whole-cell imaging at nanometer resolutions using fast and slow focused helium ions. Biophys. J. 101, 1788-1793 (2011).

29. Chen, X. et al. High-resolution 3D imaging and quantification of gold nanoparticles in a whole cell using scanning transmission ion microscopy. Biophys. J. 104, 1419-1425 (2013).

30. Udalagama, C., Bettiol, A. A. \& Watt, F. Stochastic spatial energy deposition profiles for $\mathrm{MeV}$ protons and $\mathrm{keV}$ electrons. Phys. Rev. B 80, 224107 (2009).

31. Udalagama, C. et al. An automatic beam focusing system for $\mathrm{MeV}$ protons. Nucl. Instrum. Methods Phys. Res. B 231, 389-393 (2005).

32. Tringe, J. W. et al. Radiation damage mechanisms for luminescence in Eu-doped GaN. J. Appl. Phys. 101, 054902 (2007).

33. Boden, S. A., Franklin, T. M., Scipioni, L., Bagnall, D. M. \& Rutt, H. N. Ionoluminescence in the helium ion microscope. Microsc. Microanal. 18 1253-1262 (2012).

34. Liu, J.-N. et al. Simultaneous nuclear imaging and intranuclear drug delivery by nuclear-targeted multifunctional upconversion nanoprobes. Biomaterials 33, 7282-7290 (2012).

35. Deng, K. et al. Aptamer-mediated up-conversion core/MOF shell nanocomposites for targeted drug delivery and cell imaging. Sci. Rep. 5, 7851 (2015).

36. Wang, F., Deng, R. \& Liu, X. Preparation of core-shell $\mathrm{NaGdF}_{4}$ nanoparticles doped with luminescent lanthanide ions to be used as upconversion-based probes. Nat. Protoc. 9, 1634-1644 (2014).
37. Bettiol, A. A., Udalagama, C. \& Watt, F. A new data acquisition and imaging system for nuclear microscopy based on a field programmable gate array card. Nucl. Instrum. Methods Phys. Res. B 267, 2069-2072 (2009).

\section{Acknowledgements}

The work was supported by the Singapore Ministry of Education through Academic Research Fund Tier 2 grant (Grant No. R144-000-306-112) and the Agency for Science, Technology and Research (A*STAR) (Grant No. 122-PSE-0014, 1231AFG028). We are grateful to R. Deng and Y. Tao for technical assistance.

\section{Author contributions}

F.W., X.L. and A.A.B. conceived and supervised the project. Z.M., Y.Z., S.K.V. and H.Q.T. designed the experiments. Z.M. and S.K.V. carried out the ion-beam imaging and spectral measurements. Z.M. and Y.Z. performed the photoluminescence imaging experiment. Y.Z. performed the nanocrystal synthesis. C.-B.C. prepared the cells. Z.M. and X.L. wrote the manuscript with input from the other authors.

\section{Additional information}

Supplementary Information accompanies this paper at http://www.nature.com/ naturecommunications

Competing financial interests: The authors declare no competing financial interests.

Reprints and permission information is available online at http://npg.nature.com/ reprintsandpermissions/

How to cite this article: Mi, Z. et al. Subwavelength imaging through ion-beam-induced upconversion. Nat. Commun. 6:8832 doi: 10.1038/ncomms9832 (2015).

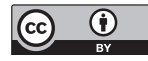

This work is licensed under a Creative Commons Attribution 4.0 International License. The images or other third party material in this article are included in the article's Creative Commons license, unless indicated otherwise in the credit line; if the material is not included under the Creative Commons license, users will need to obtain permission from the license holder to reproduce the material. To view a copy of this license, visit http://creativecommons.org/licenses/by/4.0/ 